\title{
Impulse Oscillometry and Spirometry Small-Airway Parameters in Mild to Moderate Bronchiectasis
}

\author{
Wei-jie Guan PhD, Jing-jing Yuan MB, Yong-hua Gao PhD, Hui-min Li MT, Jin-ping Zheng MD, \\ Rong-chang Chen MD, and Nan-shan Zhong MD
}

\begin{abstract}
BACKGROUND: Both impulse oscillometry and spirometry can reflect small-airway disorders. The objective of this work was to investigate the diagnostic value of impulse oscillometry and spirometry small-airway parameters and their correlation with radiology, disease severity, and sputum bacteriology in mild to moderate bronchiectasis (bronchiectasis severity index $<9$ ) and to validate these findings in sensitivity analyses (mild bronchiectasis). METHODS: We recruited 94 subjects with mild to moderate bronchiectasis and 26 healthy subjects. The diagnostic value of small-airway parameters was compared using the receiver operating characteristic curve. Chest high-resolution computed tomography (HRCT), impulse oscillometry measurement, spirometry, and sputum culture were performed. Correlation between small-airway parameters and clinical indices was determined, adjusting for age, sex, body mass index, and smoking history. Sensitivity analyses were repeated when excluding subjects with bronchiectasis severity index $\geq 9$ or HRCT score $\geq 13$. RESULTS: Impulse oscillometry and spirometry small-airway parameters could discriminate mild to moderate bronchiectasis from healthy subjects and correlated significantly with HRCT score and the number of bronchiectatic lobes and the bronchiectasis severity index (all $P<.01)$. Small-airway parameters were more aberrant in subjects with dyshomogeneity and cystic bronchiectasis but were independent of Pseudomonas aeruginosa isolation or the location of predominant bronchiectatic lobes. Spirometry, but not impulse oscillometry, small-airway parameters differed statistically between subjects with isolated peripheral-airway bronchiectasis and those with peripheral plus central-airway bronchiectasis (all $P<.01$ ). Subgroup analyses yielded similar findings, except for the lack of correlation between small-airway parameters and clinical parameters in subjects with HRCT score $\leq 6$. CONCLUSIONS: Impulse oscillometry and spirometry small-airway parameters have similar diagnostic value in reflecting peripheral-airway disorders and correlate with the HRCT scores, the bronchiectasis severity index, and the number of bronchiectatic lobes in mild to moderate bronchiectasis. Assessment of small-airway parameters should be incorporated in future lung function investigations in bronchiectasis. Key words: bronchiectasis; small-airway parameter; impulse oscillometry; spirometry; bronchiectasis severity index. [Respir Care 2016;61(11):1513-1522. @ 2016 Daedalus Enterprises]
\end{abstract}

\section{Introduction}

Bronchiectasis is a chronic debilitating airway disease that affects the large and/or small airways ${ }^{1,2}$ and can be

Drs Guan, Ms Yuan, Ms Li, Dr Zheng, Dr Chen, and Dr Zhong are affiliated with the State Key Laboratory of Respiratory Disease, National Clinical Research Center for Respiratory Disease, Guangzhou Institute of Respiratory Disease, First Affiliated Hospital of Guangzhou Medical University, Guangzhou Medical University, Guangzhou, Guangdong, China. Dr Gao is affiliated with the Department of Respiratory and detected with radiological techniques, such as high-resolution computed tomography (HRCT), ${ }^{3}$ and lung function

Critical Care Medicine, First Affiliated Hospital of Zhengzhou University, Zhengzhou, Henan, China.

This work was supported by the Changjiang Scholars and Innovative Research Team in University Grant ITR0961; the National Key Technology R\&D Program of the 12th National Five-Year Development Plan Grant 2012BAI05B01; National Key Scientific and Technology Support Program, Collaborative Innovation of Clinical Research for Chronic Obstructive Pulmonary Disease and Lung Cancer, Grant 2013BAI09B09 
tests, such as spirometry, ${ }^{4-6}$ diffusing capacity, ${ }^{7}$ impulse oscillometry, ${ }^{8,9}$ and the multiple-breath nitrogen wash-out technique. ${ }^{10}$ Of lung function tests, spirometry (particularly the parameters reflecting large-airway disorders) is the mainstay assessment tool for respiratory diseases. ${ }^{4-6}$ Impulse oscillometry may reflect the airway resistance and impedance, particularly peripheral-airway abnormality in bronchiectasis. ${ }^{9}$ However, apart from the greater sensitivity in detecting earlystage airway disorders, impulse oscillometry was not superior to $\mathrm{FEV}_{1}$ for assessment of bronchiectasis. ${ }^{9,11}$

In bronchiectasis, small-airway disorders have been frequently observed yet neglected. This has been exemplified by the fact that a considerable proportion of subjects demonstrated significantly lower levels of spirometry smallairway parameters as compared with healthy subjects ${ }^{4}$ and that, according to chest HRCT, small-airway obstruction arose from mucus plugging of the lung periphery. There have been scant treatment options that would alleviate small-airway disorders in bronchiectasis, which would clinically translate into improved gas ventilation and diffusion in the lung periphery and ameliorated ventilation heterogeneity. Except for the very few etiologies contributing to predominantly central-airway bronchiectasis, small-airway disorders frequently precede large-airway disorders, with their severity being closely linked to the magnitude of

(to Drs Zhong and Chen); National Natural Science Foundation Grant 81400010; and 2014 Scientific Research Projects for Medical Doctors and Researchers from Overseas, Guangzhou Medical University, Grant 2014C21 (to Dr Guan).

Drs Zhong and Chen have disclosed relationships with the Changjiang Scholars and Innovative Research Team in University; the National Key Technology R\&D Program of the 12th National Five-Year Development Plan; and the National Key Scientific and Technology Support Program: Collaborative Innovation of Clinical Research for Chronic Obstructive Pulmonary Disease and Lung Cancer. Dr Guan has disclosed relationships with the National Natural Science Foundation and the 2014 Scientific Research Projects for Medical Doctors and Researchers from Overseas, Guangzhou Medical University. The other authors have disclosed no conflicts of interest. None of the funding sources had any role in the study.

Supplementary material related to this paper is available at http:// www.rcjournal.com.

Correspondence: Nan-shan Zhong MD, State Key Laboratory of Respiratory Disease, National Clinical Research Center for Respiratory Disease, Guangzhou Institute of Respiratory Disease, First Affiliated Hospital of Guangzhou Medical University, Guangzhou Medical University, 151 Yanjiang Road, Guangzhou, Guangdong 510120, China. E-mail: nanshan@vip.163.com. Rong-chang Chen MD, State Key Laboratory of Respiratory Disease, National Clinical Research Center for Respiratory Disease, Guangzhou Institute of Respiratory Disease, First Affiliated Hospital of Guangzhou Medical University, Guangzhou Medical University, 151 Yanjiang Road, Guangzhou, Guangdong 510120, China. E-mail: chenrc@vip.163.com.

DOI: $10.4187 /$ respcare .04710

\section{QUICK LOOK}

\section{Current knowledge}

Previous studies have shown that impulse oscillometry confers similar diagnostic performance to bronchiectasis compared with spirometry. Because most studies have focused on large-airway parameters, the usefulness of small-airway parameters for assessment of (particularly mild to moderate) bronchiectasis is unclear.

\section{What this paper contributes to our knowledge}

Spirometry and impulse oscillometry small-airway parameters could discriminate subjects with mild to moderate bronchiectasis from healthy subjects. The fact that impulse oscillometry small-airway parameters better reflect peripheral-airway disorders and that all small-airway parameters correlate with the number of bronchiectatic lobes, high-resolution computed tomography scores, and the bronchiectasis severity index has provided a scientific rationale for incorporating small-airway parameters into the assessment of lung physiology in mild to moderate bronchiectasis.

airway inflammation or remodeling. ${ }^{12-15}$ This suggests that the detection of small-airway disorders might also reflect patients' overall well-being. Impulse oscillometry parameters that detect small-airway disorders reportedly add diagnostic information to spirometry in predicting loss of asthma control. ${ }^{16}$ However, it remains unclear whether the small-airway parameters of spirometry and impulse oscillometry correlate with bronchiectasis severity in adults with bronchiectasis, particularly milder forms.

Hence, we first compared the diagnostic value of impulse oscillometry and spirometry small-airway parameters for discriminating subjects with mild to moderate bronchiectasis from healthy subjects. Next, we assessed the association between small-airway parameters and chest radiology, sputum bacteriology, and disease severity. We also determined whether impulse oscillometry and spirometry small-airway parameters differed significantly according to the sites of bronchiectatic airways. Finally, the above findings were replicated in subgroup analyses by excluding subjects with moderate bronchiectasis or an HRCT score of $\geq 13$.

\section{Methods}

\section{Subjects}

The study protocol was approved by the ethics committee of the First Affiliated Hospital of Guangzhou Medical University (approval: Medical Ethics Year 2012 [The 33rd]). All subjects gave written informed consent. 
Subjects were recruited from September 2012 to March 2015. Diagnosis of bronchiectasis was made according to chest HRCT at 2-mm collimation within 12 months and clinical symptoms of chronic coughing, purulent sputum, and/or hemoptysis. Patients $18-75$ y old who remained clinically stable (symptoms/signs not exceeding normal daily variations: cough frequency, 24-h sputum volume, sputum purulence, fever, dyspnea, and chest pain) ${ }^{4,9}$ for $\geq 4$ weeks were eligible for recruitment. We excluded patients with severe bronchiectasis (bronchiectasis severity index $\geq 9$ ), ${ }^{16}$ severe systemic diseases (uncontrolled hypertension, recent cerebral stroke, or malignancy), use of oral or systemic antibiotics within 4 weeks, or limited understanding.

A single set of healthy subjects were recruited between September 2012 and October 2013. They were 18-75 y old, had baseline $\mathrm{FEV}_{1} \geq 80 \%$ predicted, had no upper respiratory tract infection within 3 weeks, and had normal chest radiography.

\section{Study Design}

This was a cross-sectional study. The diagnostic value of impulse oscillometry and spirometry small-airway parameters in discriminating mild to moderate bronchiectasis from healthy subjects was compared, and their association with clinical parameters (including HRCT characteristics, sputum bacteriology, and previous history) was determined. To verify our findings, all analyses were replicated in sensitivity analyses that excluded subjects with moderate bronchiectasis (bronchiectasis severity index $\geq 9$ ) or those with HRCT score $\geq 7$.

\section{Impulse Oscillometry}

Impulse oscillometry measurement was conducted before spirometry, by using the JAEGER MS-IOS system (CareFusion, Hochberg, Germany). The system was calibrated each day before the measurement.

Subjects were seated upright, with their head straight and in slight extension. Subjects tightly sealed their lips around the mouthpiece and firmly supported their cheeks with both palms. A nose clip was applied, and subjects breathed tidally for 20-30 s during each maneuver. Three or more reproducible measurements that were free of artifacts (swallowing, breath-holding, glottis closure, or cough) were assessed. Readings of the maneuvers with a variation coefficient of $<10 \%$ were averaged. The smallairway parameters of impulse oscillometry included lung reactance at $5 \mathrm{~Hz}\left(\mathrm{X}_{5}\right)$, the difference between lung resistance at $5 \mathrm{~Hz}$ and $20 \mathrm{~Hz}\left(\mathrm{R}_{5}-\mathrm{R}_{20}\right)$, resonance frequency (Fres), and reactance area (AX). ${ }^{9,14,15}$ Lung resistance at 5 and $20 \mathrm{~Hz}$ was not analyzed because it primarily reflected the whole- and central-airway disorders, respectively.

\section{Spirometry}

A QUARK PFT spirometer (COSMED, Milan, Italy) was employed for spirometry assessment, in compliance with the start-of-test and end-of-test acceptability criteria as established by international guidelines. ${ }^{17}$ Results from maneuvers with artifacts, such as coughing and premature glottis closure, were discarded. Three to eight maneuvers were performed, with $<5 \%$ or $200 \mathrm{~mL}$ variation in FVC and $\mathrm{FEV}_{1}$. Maximal FVC and $\mathrm{FEV}_{1}$ were reported. Spirometry small-airway parameters included forced expiratory flow during the middle half of the $\mathrm{FVC}$ maneuver $\left(\mathrm{FEF}_{25-75 \%}\right)$, forced expiratory flow at $50 \%$ lung volume $\left(\mathrm{FEF}_{50 \%}\right)$, and forced expiratory flow at $75 \%$ lung volume $\left(\mathrm{FEF}_{75 \%}\right)$, selected from the maneuver with maximal sum of FVC and $\mathrm{FEV}_{1}$. Predicted values were recommended by Zheng and Zhong. ${ }^{18}$

\section{HRCT Scores}

The modified Reiff score was assessed on a lobar basis (including the lingular lobe). The degree of bronchiectasis was scored $(0=$ none, $1=$ tubular, $2=$ varicose, and $3=$ cystic bronchiectasis) for individual lobes. The maximal HRCT score was $18 .{ }^{19}$ Other imaging characteristics, including dyshomogeneity, cystic bronchiectasis, and predominantly lower lobe bronchiectasis, were also determined. ${ }^{9}$

\section{Bronchiectasis Severity Metrics}

The bronchiectasis severity index was a composite measure consisting of age, body mass index, prior exacerbation frequency and prior hospitalization, dyspnea score, percent-of-predicted $\mathrm{FEV}_{1}$, Pseudomonas aeruginosa infection, colonization with other pathogenic microorganisms, and the number of bronchiectatic lobes. A bronchiectasis severity index of $\leq 4,5-8$, and $\geq 9$ denoted mild, moderate, and severe bronchiectasis, respectively. ${ }^{16} \mathrm{Al}-$ though the bronchiectasis severity index might not specifically reflect the radiologic severity of bronchiectasis, it has become one of the accepted standard criteria for assessment of bronchiectasis severity; hence, the bronchiectasis severity index was also employed in this study.

\section{Sputum Culture}

See the supplementary materials at http://www. rcjournal.com.

\section{Statistical Analysis}

The sample size was estimated with G*Power 3.1.9.2 (Heinrich-Heine-Universität, Düsseldorf, Germany). ${ }^{20}$ Assuming a correlation coefficient between small-airway parameters (ie, Fres) and the bronchiectasis severity index of 


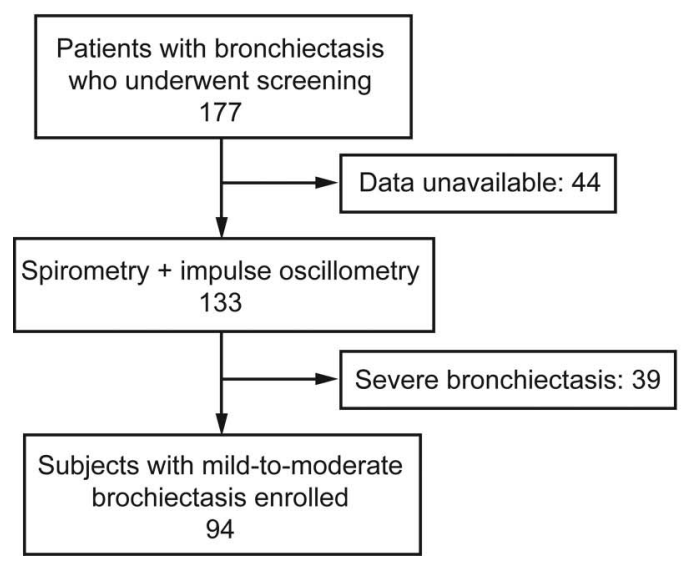

Fig. 1. Flow chart.

0.30 and a probability of error of .05 , the estimated sample size would be 88 to provide a statistical power of 0.90 .

SPSS 16.0 (SPSS, Chicago, Illinois) and GraphPad Prism 5.0 (GraphPad Software, La Jolla, California) were utilized for statistical analyses. Numerical data are presented as mean $\pm \mathrm{SD}$ or median (interquartile range) if appropriate. Two-group comparisons were made using an independent $t$ test or Mann-Whitney test, whereas 3-group comparisons used a one-way analysis of variance or Kruskal-Wallis test. Categorical variables are presented as $n(\%)$ and compared with the chi-square test. The diagnostic value of small-airway parameters was compared using the receiver operating characteristic curve, along with the area under the curve, sensitivity, and specificity. To minimize potential confounding, correlation coefficients have been adjusted for age, sex, body mass index, and smoking history. $P<.05$ denoted statistical significance.

\section{Results}

\section{Subject Enrollment}

Of 177 patients with bronchiectasis who underwent screening, 133 had performed spirometry and impulse oscillometry measurements. Of these subjects with bronchiectasis, 54 had mild and 40 had moderate bronchiectasis). Twenty-eight healthy subjects were enrolled, of whom 2 had incomplete data; therefore, 26 were included in the final analyses (Fig. 1).

\section{Subject Characteristics}

Subjects with mild to moderate bronchiectasis had similar anthropometric parameters compared with healthy subjects except for lower body mass index $(P<.01)$. Furthermore, subjects with bronchiectasis included a greater proportion of never-smokers than healthy subjects. In sub-

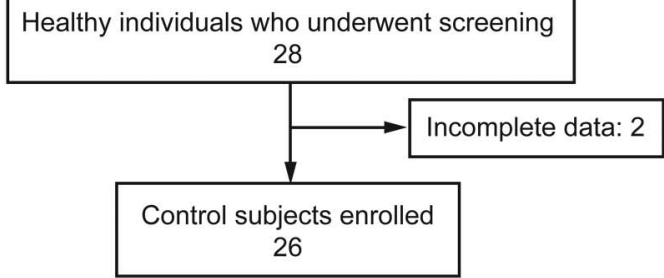

jects with bronchiectasis, the median duration of symptom onset was $4.0 \mathrm{y}$, the HRCT total score was 5.0, and the bronchiectasis severity index was 4.0 , respectively (Table 1). Percent-of-predicted $\mathrm{FEV}_{1}$ was $74.4 \pm 22.5$ and $99.9 \pm 11.1 \%$ in subjects with mild to moderate bronchiectasis and healthy subjects, respectively.

\section{Impulse Oscillometry and Spirometry Small-Airway Parameters Discriminated Subjects With Mild to Moderate Bronchiectasis From Healthy Subjects}

Compared with healthy subjects, subjects with mild to moderate bronchiectasis yielded significantly lower levels of percent-of-predicted $\mathrm{FEF}_{25-75 \%}$, percent-of-predicted $\mathrm{FEF}_{50 \%}$, percent-of-predicted $\mathrm{FEF}_{75 \%}$, and $\mathrm{X}_{5}$ and higher levels of $\mathrm{R}_{5}-\mathrm{R}_{20}$, Fres, and AX (all $P<.01$; Fig. 2).

The receiver operating characteristic curve was plotted to display the diagnostic value of different small-airway parameters (Fig. 3). Overall, small-airway parameters could discriminate subjects with mild to moderate bronchiectasis from healthy subjects. The diagnostic performance of percent-of-predicted $\mathrm{FEF}_{50 \%}$ was highest (area under the curve: 0.84), followed by percent-of-predicted $\mathrm{FEF}_{25-75 \%}$ (area under the curve: 0.80 ), percent-of-predicted $\mathrm{FEF}_{75 \%}$ (area under the curve: 0.79), Fres (area under the curve: 0.78), AX (area under the curve: 0.72 ), $R_{5}-R_{20}$ (area under the curve: 0.67 ), and $\mathrm{X}_{5}$ (area under the curve: 0.67 ). The assay sensitivity and specificity of small-airway parameters ranged from 0.37 to 0.73 and from 0.58 to 1.00 , respectively (Table 2 ).

\section{Small-Airway Parameters Stratified By Clinical Indices in Mild to Moderate Bronchiectasis}

As demonstrated in Table 3, bronchiectasis subjects with dyshomogeneity or cystic bronchiectasis yielded consistently lower percent-of-predicted $\mathrm{FEF}_{25-75 \%}$, percent-of-predicted $\mathrm{FEF}_{50}$, percent-of-predicted $\mathrm{FEF}_{75}$, and $\mathrm{X}_{5}$ and higher $\mathrm{AX}$ 
Table 1. Baseline Characteristics

\begin{tabular}{lcc}
\hline \hline \multicolumn{1}{c}{ Parameter } & $\begin{array}{c}\text { Mild to moderate } \\
\text { bronchiectasis } \\
(n=94)\end{array}$ & $\begin{array}{c}\text { Healthy } \\
\text { subjects } \\
(n=26)\end{array}$ \\
\hline Anthropometry & & \\
Age, mean \pm SD y & $43.2 \pm 13.8$ & $40.1 \pm 10.7$ \\
Height, mean \pm SD cm & $161.1 \pm 7.0$ & $163.8 \pm 9.7$ \\
Body mass index, median (IQR) kg/m ${ }^{2}$ & $20.3(18.4-23.3)$ & $22.6 \pm 3.1$ \\
Never-smokers, $n$ (\%) & $84(89.4)$ & $19(73.0)$ \\
Disease characteristics, median (IQR) & & \\
Duration of symptoms, y & $10.0(5.0-20.0)$ & NA \\
No. of exacerbations within 2 years & $3.0(1.0-5.0)$ & NA \\
No. of bronchiectatic lobes & $3.0(2.0-5.0)$ & NA \\
HRCT total score & $5.0(3.8-9.0)$ & NA \\
Bronchiectasis severity index & $4.0(2.0-6.0)$ & NA \\
Bronchiectasis etiology, $n(\%) *$ & & \\
Idiopathic & $41(43.6)$ & NA \\
Post-infectious & $30(31.9)$ & NA \\
Immunodeficiency & $12(12.8)$ & NA \\
Other known etiologies $\dagger$ & $14(14.9)$ & NA \\
Sputum bacteriology, $n(\%)$ & & \\
Pseudomonas aeruginosa & $22(23.4)$ & NA \\
Haemophilus influenzae & $8(8.5)$ & NA \\
Other pathogenic bacteria $\neq$ & $20(21.3)$ & NA \\
Commensals & $44(46.8)$ & NA \\
Medications ever used within & & \\
6 months, $n$ (\%) & $70(74.5)$ & NA \\
Mucolytics & $36(38.3)$ & NA \\
Macrolides & $16(17.0)$ & NA \\
Inhaled corticosteroids & &
\end{tabular}

No subjects were using inhaled or oral antibiotics at the time of the study.

* A minor proportion of subjects were deemed as having dual etiologies; therefore, the total percentage of individual etiology slightly exceeded $100 \%$.

$\dagger$ Other known etiologies included rheumatoid arthritis, gastroesophageal reflux disease,

asthma (clinically stable), COPD, yellow nail syndrome, lung sequestration syndrome, and aspergillosis.

‡ Other pathogenic bacteria: potentially pathogenic microorganisms. In our study, other potentially pathogenic microorganisms consisted of Haemophilus parainfluenzae,

Staphylococcus aureus, Klebsiella species, Stenotrophomonas maltophilia, Escherichia coli, Sphingomonas paucimobilis, Alcaligenes faecalis subspecies faecalis, Pseudomonas

pseudoalcaligenes, and Serratia marcescens.

$\mathrm{IQR}=$ interquartile range

$\mathrm{NA}=$ not applicable

HRCT $=$ high-resolution computed tomography
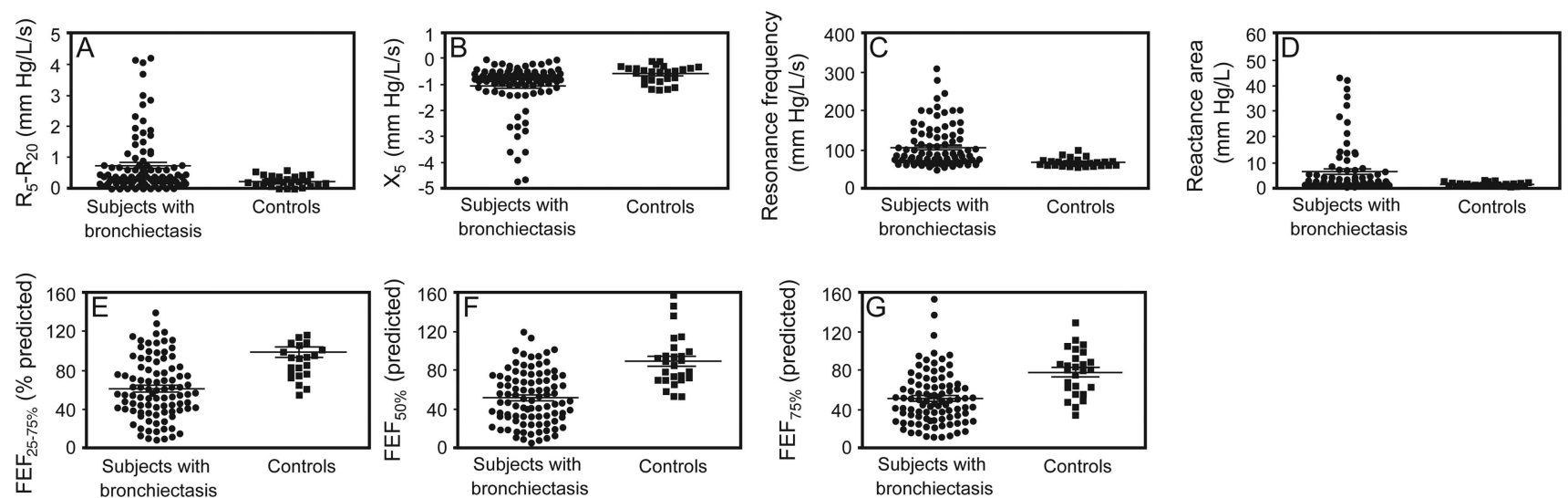

(all $P<.05$ ). Fres was significantly higher in subjects with dyshomogeneity $(P=.036)$ but marginally higher in subjects with cystic bronchiectasis $(P=.060)$. Neither isolation of $P$. aeruginosa from sputum nor predominantly lower lobe bronchiectasis correlated with greater abnormality of the small-airway parameters in mild to moderate bronchiectasis (all $P>.05$ ). $\mathrm{R}_{5}-\mathrm{R}_{20}$ was not affected by dyshomogeneity, cystic bronchiectasis, P. aeruginosa isolation, or predominantly lower lobe bronchiectasis.

\section{Small-Airway Parameters Correlated With HRCT Characteristics in Mild to Moderate Bronchiectasis}

Small-airway parameters had a moderate correlation with the number of bronchiectatic lobes and HRCT score. $\mathrm{R}_{5^{-}}$ $\mathrm{R}_{20}, \mathrm{X}_{5}$, Fres, and AX, but not spirometry small-airway parameters, correlated statistically with the exacerbation frequency within the previous 2 y (all $P<.01$ ). Despite being statistically significant, the correlation between small-airway parameters and the bronchiectasis severity index was not as strong as the number of bronchiectatic lobes and HRCT score. There was no significant correlation between small-airway parameters and the duration of symptom onset (Table 4).

\section{Impulse Oscillometry and Spirometry Small-Airway Parameters When Stratified by the Different Sites of Bronchiectatic Airways}

To clarify whether impulse oscillometry and spirometry small-airway parameters were specific to the disorders of lung periphery in bronchiectasis, we also compared these parameters according to different sites of bronchiectatic airways. Subjects with mild to moderate bronchiectasis yielded significantly lower levels of percent-of-predicted

Fig. 2. Comparison of impulse oscillometry and spirometry small-airway parameters between subjects with mild-to-moderate bronchiectasis and controls. A: Difference in lung resistance at 5 and $20 \mathrm{~Hz}\left(\mathrm{R}_{5}-\mathrm{R}_{20}\right)$. B: Lung reactance at $5 \mathrm{~Hz}\left(\mathrm{X}_{5}\right)$. C: Resonance frequency. D: Reactance area. E: Percentage-of-predicted mid-expiratory flow (MMEF). F: Percentage-of-predicted mid-expiratory flow at 50\% lung volume $\left(\mathrm{MEF}_{50 \%}\right)$. G: Percentage-of-predicted mid-expiratory flow at $25 \%$ lung volume $\left(\mathrm{MEF}_{25 \%}\right)$. $P<.01$ between groups for all panels. 

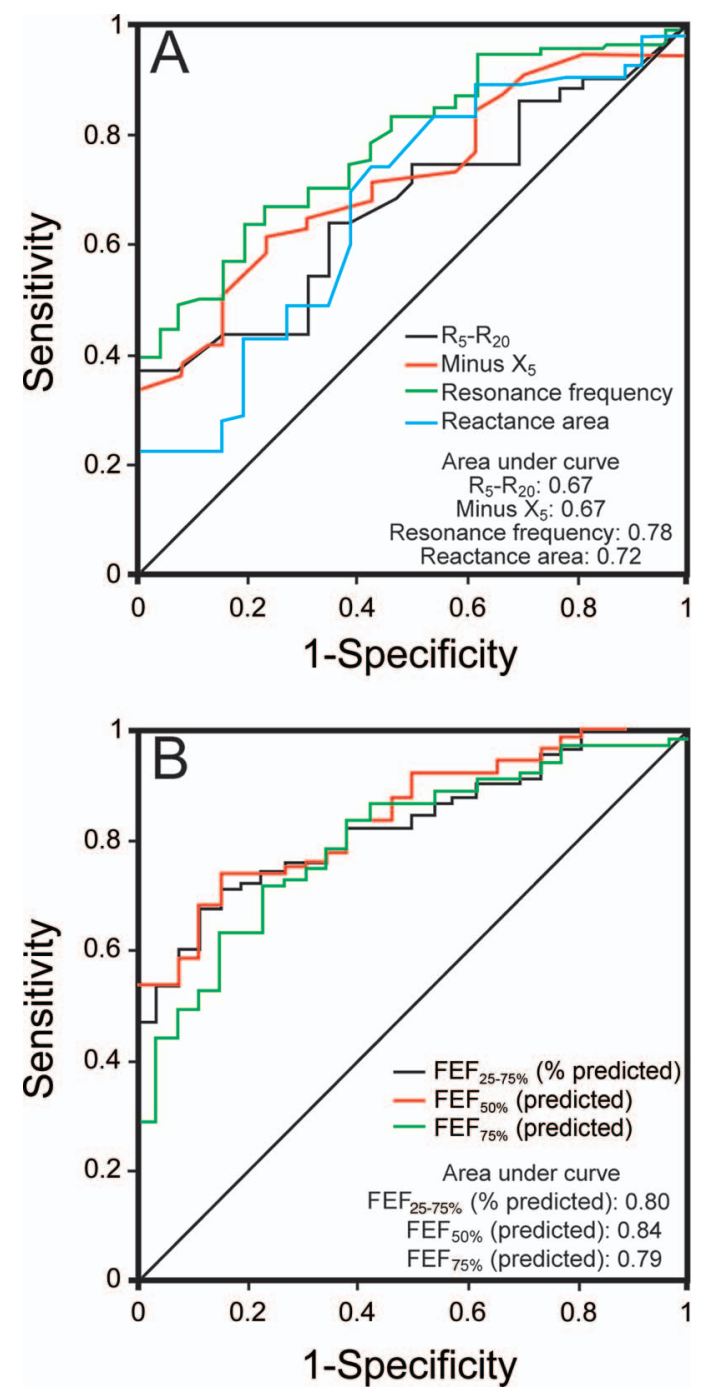

Fig. 3. Diagnostic performance of impulse oscillometry and spirometry small-airway parameters in discriminating subjects with mild-to-moderate bronchiectasis from controls.

$\mathrm{FEF}_{25-75 \%}$, percent-of-predicted $\mathrm{FEF}_{50 \%}$, percent-of-predicted $\mathrm{FEF}_{75 \%}$, and $\mathrm{X}_{5}$ and higher levels of $\mathrm{R}_{5}-\mathrm{R}_{20}$, Fres, and AX (all $P<.05$ ) than healthy subjects; therefore, small-airway disorders could explain the lung function abnormalities in subjects with isolated peripheral-airway bronchiectasis. We found that spirometry, but not impulse oscillometry, small-airway parameters discriminated subjects with isolated peripheral-airway bronchiectasis from those with peripheral plus central-airway bronchiectasis (see the supplementary table 1 at http://www.rcjournal.com).

\section{Sensitivity Analysis: Subjects With Mild Bronchiectasis}

Next, we replicated the analyses in subjects with mild bronchiectasis (bronchiectasis severity index: 0-4). As expected, lower levels of percent-of-predicted $\mathrm{FEF}_{25-75 \%}$, percent-of-predicted $\mathrm{FEF}_{50}$, percent-of-predicted $\mathrm{FEF}_{75}$, and $X_{5}$ and higher levels of $R_{5}-R_{20}$, Fres, and $A X$ were noted in subjects with mild bronchiectasis (data not shown). The receiver operating characteristic curve demonstrated similar findings compared with all subjects with mild to moderate bronchiectasis, except that areas under the curve were slightly lower. Percent-of-predicted $\mathrm{FEF}_{50}$ and $\mathrm{FEF}_{25-75 \%}$ had the highest diagnostic value, followed by percent-of-predicted $\mathrm{FEF}_{75}$, Fres, $\mathrm{AX}$, and $\mathrm{X}_{5}$ (see the supplementary table 2 and supplementary figure 1 ).

Subjects with cystic bronchiectasis had markedly lower percent-of-predicted $\mathrm{FEF}_{25-75 \%}$, percent-of-predicted $\mathrm{FEF}_{50 \%}$, and $\mathrm{X}_{5}$ and higher levels of $\mathrm{R}_{5}-\mathrm{R}_{20}$. However, the levels of percent-of-predicted $\mathrm{FEF}_{75 \%}$ $(P=.069)$, Fres, and AX $(P=.061)$ marginally differed. Subjects with dyshomogeneity yielded significantly lower percent-of-predicted $\mathrm{FEF}_{50 \%}(P=.036)$ yet marginally lower percent-of-predicted $\mathrm{FEF}_{25-75 \%}$ $(P=.050)$. Neither $P$. aeruginosa isolation nor predominantly middle/lower bronchiectasis significantly modified the impulse oscillometry or spirometry smallairway parameters (see the supplementary table 3 ).

Small-airway parameters had moderate correlations with HRCT total score, the number of bronchiectatic lobes, and the bronchiectasis severity index. Neither impulse oscillometry nor spirometry small-airway parameters correlated with the duration of symptom onset or exacerbation frequency within 2 y (all $P>.05$; see the supplementary table 4). Impulse oscillometry, but not spirometry, small-airway parameters were comparable between subjects with isolated peripheral-airway bronchiectasis and those with peripheral plus centralairway bronchiectasis (all $P>.05$; data not shown).

\section{Sensitivity Analysis: Subjects With HRCT Score $\leq 6$}

Finally, we replicated analyses in subjects with HRCT scores of $\leq 6$ (radiologically mild bronchiectasis). Subjects with bronchiectasis yielded lower levels of percent-of-predicted $\mathrm{FEF}_{25-75 \%}$, percent-of-predicted $\mathrm{FEF}_{50 \%}$, percentof-predicted $\mathrm{FEF}_{75 \%}$, and $\mathrm{X}_{5}$ and higher levels of $\mathrm{R}_{5}-\mathrm{R}_{20}$, Fres, and AX compared with healthy subjects (data not shown).

Findings of the receiver operating characteristic curve mirrored those of the whole bronchiectasis cohort, except for the numerically lower areas under the curve. Percentof-predicted $\mathrm{FEF}_{50 \%}$ yielded the highest diagnostic value, followed by percent-of-predicted $\mathrm{FEF}_{25-75 \%}$, percent-ofpredicted $\mathrm{FEF}_{75 \%}$, Fres, $\mathrm{AX}, \mathrm{X}_{5}$, and $\mathrm{R}_{5}-\mathrm{R}_{20}$ (see the supplementary table 5 and supplementary figure 2 ). Nonetheless, impulse oscillometry and spirometry small-airway parameters were not influenced by the location of predominant bronchiectatic lobes, $P$. aeruginosa isolation, dyshomogeneity, or cystic bronchiectasis (all $P>.05$; Table 
Table 2. Diagnostic Value of Impulse Oscillometry and Spirometry Small-Airway Parameters to Discriminate Subjects With Mild to Moderate Bronchiectasis From Healthy Subjects

\begin{tabular}{|c|c|c|c|c|c|c|}
\hline Parameters & Area under curve & $P$ & $95 \% \mathrm{CI}$ & Cutoff & Sensitivity & Specificity \\
\hline $\mathrm{FEF}_{25-75 \%}, \%$ predicted & 0.80 & $<.001$ & $0.74-0.90$ & 72.0 & 0.67 & 0.89 \\
\hline $\mathrm{FEF}_{50 \%}, \%$ predicted & 0.84 & $<.001$ & $0.76-0.91$ & 70.1 & 0.73 & 0.85 \\
\hline $\mathrm{FEF}_{75 \%}, \%$ predicted & 0.79 & $<.001$ & $0.70-0.88$ & 60.3 & 0.71 & 0.77 \\
\hline $\mathrm{R}_{5}-\mathrm{R}_{20}(\mathrm{~mm} \mathrm{Hg} / \mathrm{L} / \mathrm{s})$ & 0.67 & $<.001$ & $0.57-0.78$ & 0.60 & 0.37 & 1.00 \\
\hline $\mathrm{X}_{5}(\mathrm{~mm} \mathrm{Hg} / \mathrm{L} / \mathrm{s})$ & 0.67 & $<.001$ & $0.56-0.79$ & -0.60 & 0.75 & 0.58 \\
\hline Fres $(\mathrm{mm} \mathrm{Hg} / \mathrm{L} / \mathrm{s})$ & 0.78 & $<.001$ & $0.69-0.87$ & 72.5 & 0.64 & 0.81 \\
\hline $\mathrm{AX}(\mathrm{mm} \mathrm{Hg} / \mathrm{L})$ & 0.72 & $<.001$ & $0.62-0.82$ & 2.93 & 0.62 & 0.77 \\
\hline
\end{tabular}

The BSI $(\leq 8)$ served as the accepted standard, whereas healthy subjects served as the control group.

$\mathrm{FEF}_{25-75 \%}=$ forced expiratory flow during the middle half of the FVC maneuver

$\mathrm{FEF}_{50}=$ forced expiratory flow at $50 \%$ lung volume

$\mathrm{FEF}_{25}=$ forced expiratory flow at $25 \%$ lung volume

$\mathrm{R}_{5}-\mathrm{R}_{20}=$ difference between lung resistance at 5 and $20 \mathrm{~Hz}$

$\mathrm{X}_{5}=$ lung reactance at $5 \mathrm{~Hz}$

Fres $=$ resonance frequency

$\mathrm{AX}=$ reactance area

S6). Overall, impulse oscillometry and spirometry smallairway parameters did not correlate statistically with the number of bronchiectatic lobes, duration of symptom onset, bronchiectasis severity index, or exacerbation frequency within 2 y (all $P>.05$; Table S7). There were no statistical differences in impulse oscillometry, but not spirometry, small-airway parameters between subjects with isolated peripheral-airway bronchiectasis and those with peripheral plus central-airway bronchiectasis (all $P>.05$; data not shown).

\section{Discussion}

\section{Principal Findings}

This study extended our previous findings ${ }^{9}$ by demonstrating that impulse oscillometry and spirometry smallairway parameters had similar diagnostic value and could discriminate subjects with mild to moderate bronchiectasis from healthy subjects, that these parameters could be affected by dyshomogeneity and cystic bronchiectasis but were unlikely to be influenced by the location of predominant bronchiectatic lobes or sputum bacteriology, and that disorders of the lung periphery accounted significantly for the abnormalities of small-airway parameters. Furthermore, correlations between small-airway parameters and clinical parameters were significant only when stratifying subjects according to the bronchiectasis severity index but not radiological scores.

\section{Interpretation and Clinical Importance}

Currently, reports comparing impulse oscillometry and spirometry in respiratory infection are scarce. Raj et al ${ }^{21}$ found that, in 34 children and 5 adults with cystic fibrosis,
AX correlated negatively with $\mathrm{FEV}_{1}$ and peak expiratory flow. However, AX conferred limited significance for identifying air flow limitation compared with $\mathrm{X}_{5}$ and $\mathrm{R}_{5}$, which reflected the whole respiratory tract. Moreau et $\mathrm{al}^{22}$ documented the inverse associations between Fres and spirometry parameters and the positive associations between $\mathrm{X}_{5}$ and spirometry parameters in cystic fibrosis children. Unfortunately, no acceptable cut-points of Fres or $\mathrm{X}_{5}$ could be established for detecting children with lung function impairment. Furthermore, neither Fres nor $\mathrm{X}_{5}$ was parallel to $\mathrm{FEV}_{1}$ reduction during follow-up. In our study, impulse oscillometry and spirometry small-airway parameters readily discriminated mild to moderate bronchiectasis, most of which had evidence of air flow limitation. Due to different study design and population, direct comparisons with these literature reports were not evaluated.

Nonetheless, AX conferred additional information to predict long-term asthma control. ${ }^{23}$ Intriguingly, although large- and small-airway parameters correlated poorly with asthma symptoms, impulse oscillometry and spirometry small-airway parameters contributed independently to the clinical expression of asthma. ${ }^{24}$ These collectively implied that small-airway parameters, when measured in conjunction with large-airway parameters of spirometry or impulse oscillometry, might have aided the assessment of respiratory diseases. Notably, small-airway disorders already existed in mild bronchiectasis. Sensitivity analyses that excluded subjects with a bronchiectasis severity index $\geq 9$ or an HRCT score of $\geq 7$, coupled with our comparisons between subjects with isolated peripheral-airway bronchiectasis and those with peripheral plus central-airway bronchiectasis, reaffirmed this finding. This is important because mild bronchiectasis has already been characterized by small-airway disorders that could be readily detected with spirometry and impulse oscillometry, nonin- 


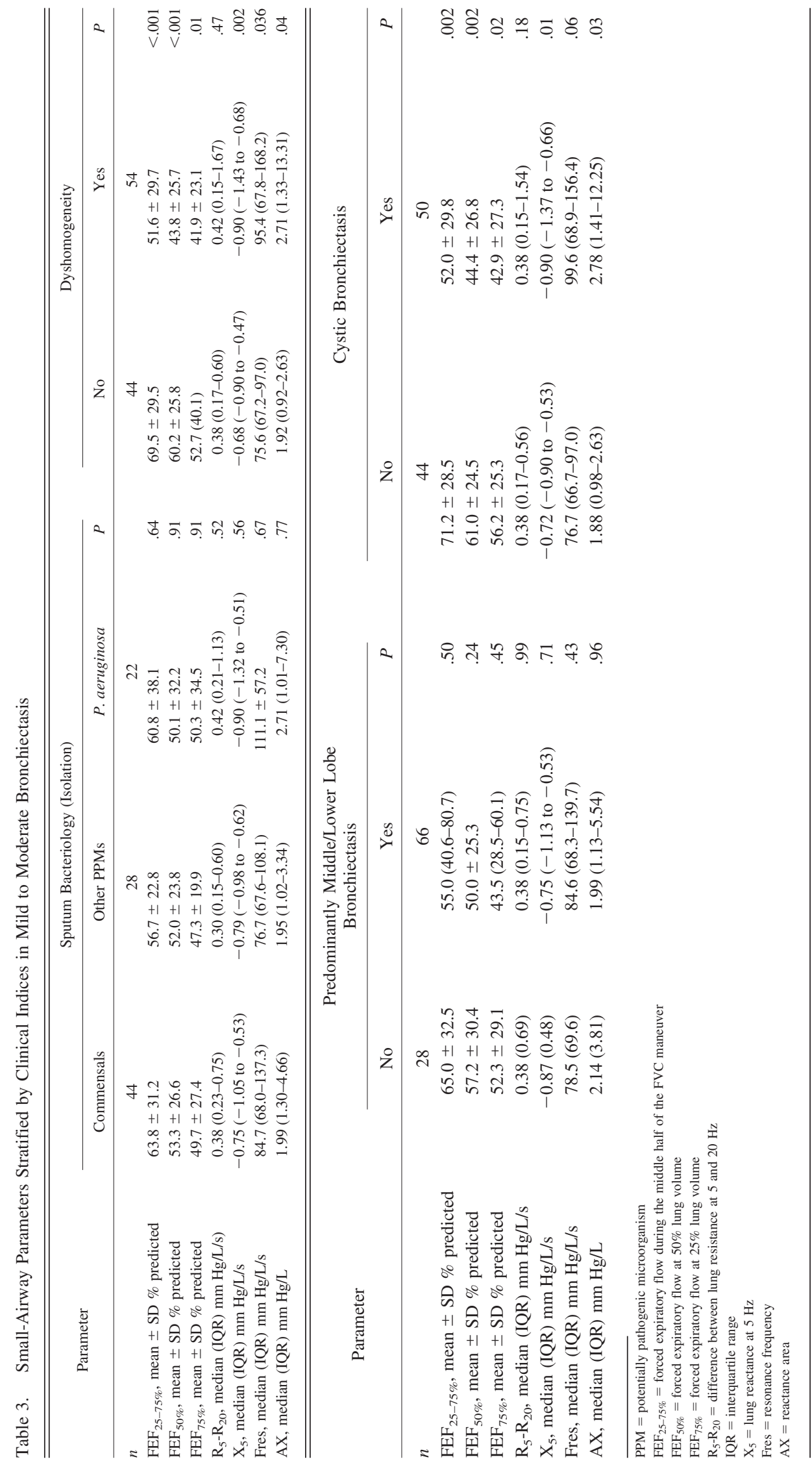


Table 4. Correlation Between Impulse Oscillometry and Spirometry Small-Airway Parameters and Clinical Parameters in Subjects With Mild to Moderate Bronchiectasis

\begin{tabular}{|c|c|c|c|c|c|c|c|c|c|c|}
\hline \multirow{2}{*}{ Parameters } & \multicolumn{2}{|c|}{$\begin{array}{c}\text { Disease } \\
\text { Duration (y) }\end{array}$} & \multicolumn{2}{|c|}{ BSI } & \multicolumn{2}{|c|}{$\begin{array}{l}\text { Bronchiectatic } \\
\text { Lobes }\end{array}$} & \multicolumn{2}{|c|}{$\begin{array}{c}\text { Exacerbations } \\
\text { Within } 2 \text { y }\end{array}$} & \multicolumn{2}{|c|}{ HRCT Score } \\
\hline & $\mathrm{r}$ & $P$ & $\mathrm{r}$ & $P$ & $\mathrm{r}$ & $P$ & $\mathrm{r}$ & $P$ & $\mathrm{r}$ & $P$ \\
\hline $\mathrm{FEF}_{25-75 \%}, \%$ predicted & -0.19 & .08 & -0.33 & .002 & -0.55 & $<.001$ & -0.15 & .16 & -0.58 & $<.001$ \\
\hline $\mathrm{FEF}_{50 \%}, \%$ predicted & -0.18 & .08 & -0.36 & $<.001$ & -0.55 & $<.001$ & -0.14 & .20 & -0.58 & $<.001$ \\
\hline $\mathrm{FEF}_{75 \%}, \%$ predicted & -0.18 & .08 & -0.32 & .002 & -0.53 & $<.001$ & -0.15 & .15 & -0.55 & $<.001$ \\
\hline $\mathrm{R}_{5}-\mathrm{R}_{20}, \mathrm{~mm} \mathrm{Hg} / \mathrm{L} / \mathrm{s}$ & 0.11 & .29 & 0.32 & .003 & 0.41 & $<.001$ & 0.35 & .001 & 0.48 & $<.001$ \\
\hline $\mathrm{X}_{5}, \mathrm{~mm} \mathrm{Hg} / \mathrm{L} / \mathrm{s}$ & -0.13 & .22 & -0.35 & .001 & -0.45 & $<.001$ & -0.34 & .001 & -0.55 & $<.001$ \\
\hline Fres, $\mathrm{mm} \mathrm{Hg} / \mathrm{L} / \mathrm{s}$ & 0.16 & .13 & 0.31 & .003 & 0.48 & $<.001$ & 0.30 & .004 & 0.50 & $<.001$ \\
\hline $\mathrm{AX}, \mathrm{mm} \mathrm{Hg} / \mathrm{L}$ & 0.06 & .59 & 0.32 & .002 & 0.40 & $<.001$ & 0.33 & .002 & 0.46 & $<.001$ \\
\hline $\begin{array}{l}\text { All } \mathrm{r} \text { values denoted the Spearma } \\
\mathrm{BSI}=\text { bronchiectasis severity ind } \\
\mathrm{HRCT}=\text { high-resolution comput } \\
\mathrm{FEF}_{25-75 \%}=\text { forced expiratory fl} \\
\mathrm{FEF}_{50 \%}=\text { forced expiratory flow } \\
\mathrm{FEF}_{75 \%}=\text { forced expiratory flow } \\
\mathrm{R}_{5}-\mathrm{R}_{20}=\text { difference between lun } \\
\mathrm{X}_{5}=\text { lung reactance at } 5 \mathrm{~Hz} \\
\text { Fres = resonance frequency } \\
\mathrm{AX}=\text { reactance area }\end{array}$ & $\begin{array}{l}\text { orelation co } \\
\text { nography } \\
\text { ring the mi } \\
\% \text { lung vol } \\
\text { \% lung volt } \\
\text { tance at } 5\end{array}$ & tsts, fol & adjustmen & the age, sex & $y$-mass inde & smoking st & & & & \\
\hline
\end{tabular}

vasive approaches without risks of exposure to radiation. Furthermore, small-airway parameters correlated most significantly with radiological severity of mild to moderate bronchiectasis. This indicated that small-airway parameters reflected small-airway disorders but remained relatively independent of the disease severity metrics, which was inconsistent with large-airway parameters such as $\mathrm{FEV}_{1}$ and airway resistance measured at $20 \mathrm{~Hz} .{ }^{9}$ Hence, measurement of small-airway parameters might offer the unique window to view the clinically silent zones beyond the scope of HRCT.

Although small-airway parameters yielded similar diagnostic value for mild to moderate bronchiectasis, spirometry (particularly $\mathrm{FEF}_{25-75 \%}$ and $\mathrm{FEF}_{50 \%}$ ) performed slightly better than impulse oscillometry. However, this may not rule out the usefulness of impulse oscillometry. In our previous study, impulse oscillometry parameters more sensitively detected mild bronchiectasis, particularly in subjects with normal lung function as assessed with spirometry. ${ }^{9}$ Measurements of frequency dependence (Fres and $\mathrm{AX}$ ), the major impulse oscillometry parameters, were less affected by the total lung capacity and therefore might more accurately reflect peripheral lung disorders. This might provide a rationale for future clinical trials to investigate the effects of superfine particles of antibiotics or corticosteroids on the airway periphery of bronchiectasis, a region that has received little attention to date.

Our findings emphasize the importance of physician vigilance to systematically evaluate functional impairment of lung periphery in mild to moderate bronchiectasis. Patients may benefit from inhaled medications (ie, extra-fine particles) that target small airways, although this awaits verification by further studies.

\section{Strengths and Limitations}

We have systematically compared the usefulness of impulse oscillometry and spirometry small-airway parameters for assessment of mild to moderate bronchiectasis. The strengths of this study include head to head comparisons and the subgroup analyses with different inclusion criteria. Nonetheless, the limited sample sizes and monocentric study design might have restricted the generalizability of our findings. Correlation analyses could have been influenced by other confounding factors that have not been adjusted. Furthermore, small-airway parameters of impulse oscillometry at inspiratory and expiratory phases were averaged. Inspiratory impulse oscillometry parameters better reflect small-airway disorders of asthma, COPD, ${ }^{25}$ and interstitial lung disease, ${ }^{26}$ which might still apply in bronchiectasis. Finally, because of the relatively short follow-up period, we could not test whether the smallairway parameters could predict future risks of exacerbations.

\section{Conclusions}

Impulse oscillometry and spirometry small-airway parameters have similar diagnostic value and correlate with radiologic severity of mild to moderate bronchiectasis. Small-airway disorders exist in mild bronchiectasis. Further studies investigating the roles of small-airway param- 


\section{Small-Airway Parameters in Mild to Moderate Bronchiectasis}

eters to predict future risks of bronchiectasis exacerbations will be of merit.

\section{ACKNOWLEDGMENTS}

We wholeheartedly thank Prof Yan Tang (State Key Laboratory of Respiratory Disease, National Clinical Research Center for Respiratory Disease, Guangzhou Institute of Respiratory Disease, First Affiliated Hospital of Guangzhou Medical University, Guangzhou Medical University) and Gang Xu (Guangzhou First People's Hospital) for assistance with patient recruitment.

\section{REFERENCES}

1. Pasteur MC, Bilton D, Hill AT, British Thoracic Society Bronchiectasis non-CF Guideline Group. British Thoracic Society guidelines for non-CF bronchiectasis. Thorax 2010;65(Suppl 1):i1-i58.

2. Barker AF. Bronchiectasis. N Engl J Med 2002;346(18):1383-1393.

3. Ooi GC, Khong PL, Chan-Yeung M, Ho JC, Chan PK, Lee JC, Lan WK, Tsang KW. High-resolution CT quantification of bronchiectasis: clinical and functional correlation. Radiology 2002;225(3):663672.

4. Guan WJ, Gao YH, Xu G, Lin ZY, Tang Y, Li HM, et al. Characterization of lung function impairment in adults with bronchiectasis. Plos One 2014;9(11):e113373.

5. Evans SA, Turner SM, Bosch BJ, Hardy CC, Woodhead MA. Lung function in bronchiectasis: the influence of Pseudomonas aeruginosa. Eur Respir J 1996;9(8):1601-1604.

6. Davies G, Wells AU, Doffman S, Watanabe S, Wilson R. The effect of Pseudomonas aeruginosa on pulmonary function in patients with bronchiectasis. Eur Respir J 2006;28(5):974-979.

7. King PT, Holdsworth SR, Freezer NJ, Villanueva E, Farmer MW, Guy P, Holmes PW. Lung diffusing capacity in adult bronchiectasis: a longitudinal study. Respir Care 2010;55(12):1686-1692.

8. Hamakawa H, Sakai H, Takahashi A, Bando T, Date H. Multifrequency oscillation technique using impulse oscillations: can it give mechanical information about the lung periphery? Adv Exp Med Biol 2013;765:73-79.

9. Guan WJ, Gao YH, Xu G, Lin ZY, Tang Y, Lin ZM, et al. Impulse oscillometry in adults with bronchiectasis. Ann Am Thorac Soc 2015; 12(5):657-665.

10. Rowan SA, Bradley JM, Bradbury I, Lawson J, Lynch T, Gustafsson $\mathrm{P}$, et al. Lung clearance index is a repeatable and sensitive indicator of radiological changes in bronchiectasis. Am J Respir Crit Care Med 2014;189(5):586-592.

11. Philley JV, Griffith DE. Impulse oscillometry and bronchiectasis. We still haven't found what we're looking for? Ann Am Thorac Soc 2015;12(5):621-622.
12. Verbanck S, Schuermans D, Vincken W. Inflammation and airway function in the lung periphery of patients with stable asthma. J Allergy Clin Immunol 2010;125(3):611-616.

13. Thompson BR, Douglass JA, Ellis MJ, Kelly VJ, O'Hehir RE, King GG, Verbanck S. Peripheral lung function in patients with stable and unstable asthma. J Allergy Clin Immunol 2013;131(5):1322-1328.

14. Shi Y, Aledia AS, Galant SP, George SC. Peripheral airway impairment measured by impulse oscillometry predicts loss of asthma control in children. J Allergy Clin Immunol 2013;131(3):718-723.

15. Shi Y, Aledia AS, Tatavoosian AV, Vijayalakshmi S, Galant SP, George SC. Relating small airways to asthma control by using impulse oscillometry in children. J Allergy Clin Immunol 2012;129(3): 671-678.

16. Chalmers JD, Goeminne P, Aliberti S, McDonnell MJ, Lonni S, Davidson J, et al. The bronchiectasis severity index: an international derivation and validation study. Am J Respir Crit Care Med 2014; 189(5):576-585.

17. Miller MR, Hankinson J, Brusasco V, Burgos F, Casaburi R, Coates A, et al. ATS/ERS task force: standardization of spirometry. Eur Respir J 2005;26(2):319-338.

18. Zheng J, Zhong N. Normative values of pulmonary function testing in Chinese adults. Chin Med J 2002;115(1):50-54.

19. Pasteur MC, Helliwell SM, Houghton SJ, Webb SC, Foweraker JE, Coulden RA, et al. An investigation into causative factors in patients with bronchiectasis. Am J Respir Crit Care Med 2000;162(4 Pt 1): 1277-1284.

20. Faul F, Erdfelder E, Lang AG, Buchner A. G*Power 3: a flexible statistical power analysis program for the social, behavioral, and biomedical sciences. Behav Res Methods 2007;39(2):175-191.

21. Raj D, Sharma GK, Lodha R, Kabra SK. Correlation between impulse oscillometry and spirometry parameters in patients with cystic fibrosis. Chron Respir Dis 2014;11(3):139-149.

22. Moreau L, Crenesse D, Berthier F, Albertini M. Relationship between impulse oscillometry and spirometry indices in cystic fibrosis children. Acta Paediatr 2009;98(6):1019-1023.

23. Larsen GL, Morgan W, Heldt GP, Mauger DT, Boehmer SJ, Chinchilli VM, et al. Impulse oscillometry versus spirometry in a longterm study of controller therapy for pediatric asthma. J Allergy Clin Immunol 2009;123(4):861-867.e1.

24. van der Wiel E, Postma DS, van der Molen T, Schiphof-Godart L, Ten Hacken NH, van den Berge M. Effects of small airway dysfunction on the clinical expression of asthma: a focus on asthma symptoms and bronchial hyperresponsiveness. Allergy 2014;69(12):1681-1688.

25. Paredi P, Goldman M, Alamen A, Ausin P, Usmani OS, Pride NB, Barnes PJ. Comparison of inspiratory and expiratory resistance and reactance in patients with asthma and chronic obstructive pulmonary disease. Thorax 2010;65(3):263-267.

26. Sugiyama A, Hattori N, Haruta Y, Nakamura I, Nakagawa M, Miyamoto $\mathrm{S}$, et al. Characteristics of inspiratory and expiratory reactance in interstitial lung disease. Respir Med 2013;107(6):875-882. 